\title{
Ergodic mixing for turbulent drift motion
}

\author{
M. B. Isichenko \\ Fusion Research Center, The University of Texas at Austin, Austin, TX 78712 \\ N. V. Petviashvili \\ Institute for Fusion Studies, The University of Texas at Austin, Austin, TX 78712
}

February 15, 1995

\begin{abstract}
The statistical properties of the long-time chaotic two-dimensional (2D) drift motion of a charged particle in an inhomogeneous magnetic field $B(x, y)$ and a time-dependent electrostatic potential $\phi(x, y, t)$ are studied by numerical symplectic integration. For a conditionally periodic potential with two or more incommensurate frequencies, an ergodic behavior is demonstrated in which the probability density of the particle position is proportional to the magnetic field $B$. The accuracy of this prediction is found to be independent of the number $N_{\omega}$ of the incommensurate frequencies for $N_{\omega} \geq 2$.
\end{abstract}

\section{Introduction}

The ergodic behavior of Hamiltonian systems with a macroscopic number of degrees of freedom is the basis of the time-tested classical statistical mechanics, yet the fundamental understanding of the statistical properties of chaotic dynamical systems remains to be achieved. Although marked by several important works $[1,2,3,4]$, the ergodic theory of dynamical systems turned out to be a very difficult problem. A satisfactory understanding of the statistical properties of chaotic dynamical systems has only been reached for a very special class of "hyperbolic" systems [3] and some generalizations thereof $[4,5]$.

The difficulty of applying the ergodic theory to more generic physically motivated Hamiltonian systems lies in the intricate foliation of the phase space by the Kolmogorov-ArnoldMoser (KAM) invariant tori $[6,7,8]$, which introduce obstacles for the ergodic mixing. The KAM tori partition the phase space of a "one-and-a-half-degree-of-freedom" (meaning 2D phase space and a time-periodic Hamiltonian) or a two-degree-of-freedom conservative system in a way such that the phase space orbits are forever trapped between the nearest KAM surfaces, and no global mixing is possible. As the number of degrees of freedom, or the number of incommensurate frequencies in the Hamiltonian time dependence, are increased, the dimension of the KAM tori is no longer sufficient to partition the phase space by impermeable boundaries, and a global wandering ("Arnold diffusion") of each orbit becomes possible. 
However, the ergodicity of this random walk has never been demonstrated and appears to be untrue in general. Based on the remarkable accuracy of the thermodynamic consequences of the ergodic hypothesis applied to a macroscopic number of degrees of freedom, it is natural to conject that the ergodicity, understood as the uniform long-time probability density distribution in the canonical phase space constrained by applicable conservation laws, is gradually improved with the growing dimension of the phase space. Unexpectedly, we find that, in our particular model described below, the ergodic behavior is established starting from the minimum dimension, for which the Arnold diffusion is possible, and is unchanged thereafter.

The goal of this work is to study the long-time statistics of low dimensional Hamiltonian systems with varying effective dimension, which depends on the number of incommensurate frequencies. We do so in the example of the $2 \mathrm{D} \mathbf{E} \times \mathbf{B}$ drift of a charged particle in a turbulent-like electrostatic field $\mathrm{E}=-\nabla \phi(x, y, t)$ and an inhomogeneous magnetic field $\mathbf{B}=B(x, y) \widehat{\mathbf{z}}$ :

$$
\dot{\mathbf{x}}=\mathbf{v}(\mathbf{x}, t) \equiv-\frac{c \boldsymbol{\nabla} \phi(\mathbf{x}, t) \times \widehat{\mathbf{z}}}{B(\mathbf{x})}
$$

Our special interest in this model lies in the expected inhomogeneous probability density of the particle position $P(\mathbf{x}) \propto B(\mathbf{x})$, which bears important consequences for the turbulent pinch effect in tokamak plasmas $[9,10,11]$. The inhomogeneity of $P(x, y)$ is due to the noncanonicity of the variables $x$ and $y$, whereas in appropriate canonical variables the probability density is uniform (ergodic). Without changing to canonical variables, the inhomogeneity of $P(\mathbf{x})$ is seen from the nontrivial invariant measure possessed by the system (1). The Liouville (continuity) equation for (1),

$$
\partial_{t} P+\nabla \cdot(P \mathbf{v})=0,
$$

has a steady-state solution

$$
P(\mathbf{x}) \propto B(\mathbf{x}),
$$

which, in the case of an ergodic mixing, signifies the fraction of time spent by the particle in the vicinity of the given point. The remarkable property of this invariant measure is its independence of the turbulent electrostatic potential $\phi$. What does depend on $\phi$ is the quality of the ergodic, or diffusion approximation applied to the $\mathbf{E} \times \mathbf{B}$ transport (1). As demonstrated in Sec. 3 for a time-periodic potential, the presence of the stationary solution (3) does not yet warrant that this solution is the long-time probability density. Following Refs. $[9,11]$, we refer to the statistical state described by the probability distribution (3) as turbulent equipartition (TEP). We study the conditions under which the TEP distribution is realized.

The rest of the paper is organized as follows. In Sec. 2 we describe our numerical model and derive a suitable symplectic integrator $[12,13]$. In Sec. 3 we present numerical results. The results are discussed is in Sec. 4. 


\section{DISCLAIMER}

Portions of this document may be illegible in electronic image products. Images are produced from the best available original document. 


\section{The model}

In order to get better statistics, we confine the particle motion to a $2 \pi \times 2 \pi$ periodic box (a torus) and therefore choose a space periodic potential

$$
\phi(\mathbf{x}, t)=\sum_{i=1}^{4} \sin \left(\mathbf{k}_{i} \cdot \mathbf{x}-\omega_{i} t\right)
$$

whose wavevectors $\mathbf{k}_{i}$ lie at the integer nodes of the reciprocal space, namely $\mathbf{k}_{1}=(2,1)$, $\mathbf{k}_{2}=(1,2), \mathbf{k}_{3}=(-1,2)$, and $\mathbf{k}_{4}=(-2,1)$. (From now on, dimensionless variables are used.) The time dependence of the potential assumes four distinct types distinguished by the number $N_{\omega}$ of incommensurate frequencies among $\omega_{i}$. The single frequency model, $N_{\omega}=1$, has all $\omega_{i}$ commensurate, which implies a time-periodic potential. The more incommensurate frequencies there are, the more "turbulent" the potential $\phi$ is supposed to be. The frequencies for the four models are as follows:

\begin{tabular}{|c|c|c|c|c|}
\hline$N_{\omega}$ & $\omega_{1}$ & $\omega_{2}$ & $\omega_{3}$ & $\omega_{4}$ \\
\hline 1 & 1 & 2 & 2 & 1 \\
2 & 1 & 2 & $1.5^{1 / 2}$ & $6^{1 / 2}$ \\
3 & 1 & $1.25^{1 / 2}$ & $1.5^{1 / 2}$ & 2 \\
4 & 1 & $1.25^{1 / 2}$ & $1.5^{1 / 2}$ & $1.75^{1 / 2}$ \\
\hline
\end{tabular}

The set of the wavevectors $\mathbf{k}_{i}$ is the same for all four models. The magnetic field is spatially periodic and varies in magnitude between 1 and 3 :

$$
B(x, y)=2+\sin x \sin y .
$$

In order to construct a symplectic integrator for the system

$$
\dot{x}=-\partial_{y} \phi(x, y, t) / B(x, y), \quad \dot{y}=\partial_{x} \phi(x, y, t) / B(x, y),
$$

we change from the noncanonical variables $(x, y)$ to the canonical $(x, p)$, where

$$
p(x, y)=\int^{y} B\left(x, y^{\prime}\right) d y^{\prime}=2 y-\sin x \cos y .
$$

Then $(6)$ is reads:

$$
\dot{x}=\partial_{p} H, \quad \dot{p}=-\partial_{x} H,
$$

where the Hamiltonian $H(x, p, t)=-\phi(x, y(x, p), t)$. This substitution amounts to introducing the Clebsch variables for the magnetic field defined by $\mathbf{B}=\boldsymbol{\nabla} x \times \nabla p$. The canonicity of the magnetic Clebsch variables $(x, p)$ for the drift motion was derived for a more general geometry in Refs. [14, 15].

In order to ensure statistical consistency [16], we use a symplectic integrator preserving the invariant measure $d x d p=B(x, y) d x d y$ on each step of the integration. The most general symplectic time-advance mapping $\left(x_{0}, p_{0}\right) \rightarrow(x, p)$ is given by the (implicit) canonical transformation defined by an arbitrary generating function $S\left(x, p_{0}, t\right)$ :

$$
p=\partial_{x} S\left(x, p_{0}, t\right), \quad x_{0}=-\partial_{p_{0}} S\left(x, p_{0}, t\right) .
$$


If the generating function satisfied the (nonintegrable) Hamilton-Jacobi equation,

$$
\partial_{t} S+H\left(x, \partial_{x} S, t\right)=0,
$$

the integrator (9) would be exact. An approximate symplectic integrator can be obtained from an approximate solution to the Hamilton-Jacobi equation (10) by Taylor expanding the generating function:

$$
S\left(x, p_{0}, t\right)=S_{0}\left(x, p_{0}\right)+t S_{1}\left(x, p_{0}\right)+\left(t^{2} / 2\right) S_{2}\left(x, p_{0}\right)+\mathcal{O}\left(t^{3}\right) .
$$

Upon substituting expansion (11) into (10), we obtain the following second-order implicit symplectic integrator:

$$
\begin{gathered}
p=p_{0}-t H_{x}+\frac{t^{2}}{2}\left[H_{x} H_{x p}+H_{p} H_{x x}-H_{x t}\right]+\mathcal{O}\left(t^{3}\right), \\
x_{0}=x-t H_{p}+\frac{t^{2}}{2}\left[H_{p} H_{x p}+H_{x} H_{p p}-H_{p t}\right]+\mathcal{O}\left(t^{3}\right),
\end{gathered}
$$

where the subscripts of $H \equiv H\left(x, p_{0}, 0\right)$ denote differentiation. In the case of an inhomogeneous magnetic field, another source of implicitness comes from the relation between $y$ and $p$ [Eq. (7)]. Equation (13) is solved for $x$ by iteration, which always converges for sufficiently small time step $t$.

In Sec. 3 we report on the numerical results obtained with the integrator (12)-(13).

\section{The results}

In Fig. 1 we show the positions of a single particle at successive moments of time separated by $2 \pi$ for two different time dependencies of the Hamiltonian. In the periodic case $N_{\omega}=1$, the mapping is a Poincare section of a one-and-a-half-degree-of-freedom system, and the KAM partition of the $(x, y)$ plane by invariant tori is quite apparent. This partition is missing from the "more ergodic" conditionally periodic case $N_{\omega}=2$. However, in the $N_{\omega}=2$ scattered plot we observe an inhomogeneity of the particle density. We expand on this below. The measure of chaos is given by the Lyapunov exponent $\gamma$, which is measured as the stretching rate $\langle d \ln |\boldsymbol{\xi}| / d t\rangle$ of the tangential dynamics $d \boldsymbol{\xi} / d t=(\boldsymbol{\xi} \cdot \nabla) \mathbf{v}(\mathbf{x}(t), t)$. In Fig. 2 we plot the finite-time Lyapunov exponent $\gamma(t)$ for two $N_{\omega}=4$ Hamiltonians differing only by rescaling of the frequencies $\omega_{i}$. The comparison of the corresponding Lyapunov exponents qualitatively agrees with the scaling $\gamma \propto \omega^{1 / 2}$ for $k v / \omega \gg 1$ [17].

Our main results concern the particle density distribution $P(x, y)$ which is shown in Fig. 3 . The plotted densities are computed over the time $t=100$, corresponding to approximately 20 Lyapunov time scales, starting from an initially uniform particle distribution in the $(x, y)$ plane. In all cases but the time-periodic one we observe the density relaxation to the TEP distribution $P(x, y) \propto B(x, y)$ within the accuracy of $2 \%$ to $3 \%$. The accuracy is measured 

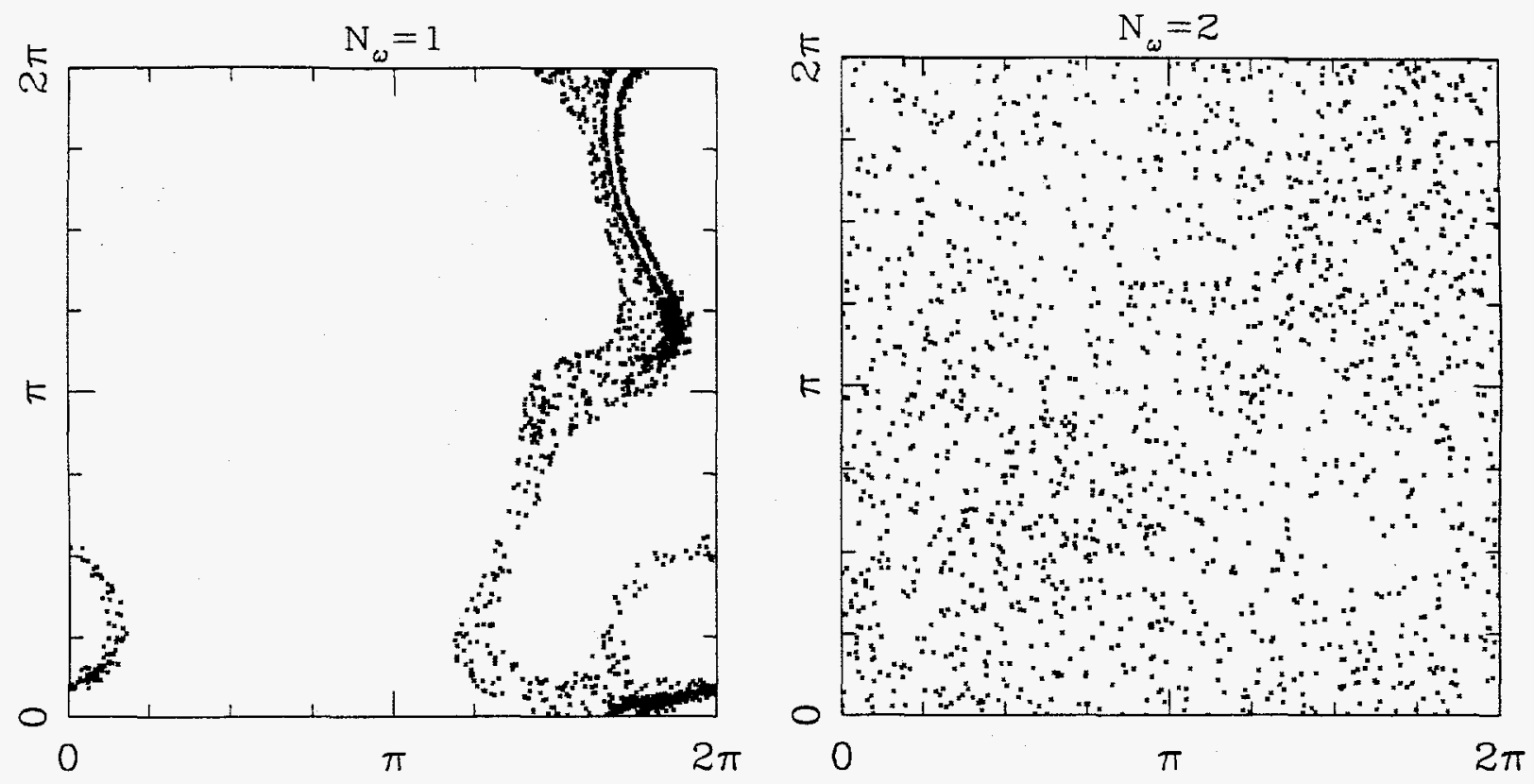

Figure 1: The $2 \pi$-time-advance mapping for a particle trajectory in the time periodic Hamiltonian $\left(N_{\omega}=1\right)$ and in the conditionally periodic Hamiltonian $\left(N_{\omega}=2\right)$.

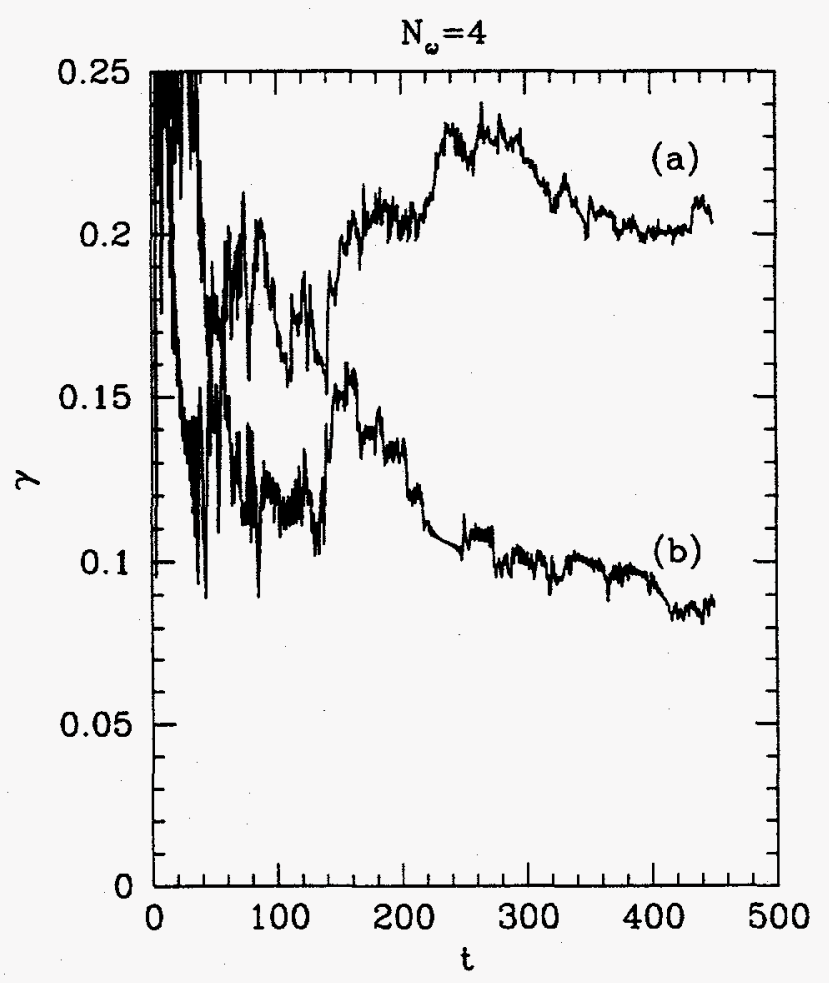

Figure 2: Finite-time Lyapunov exponents for the four-frequency Hamiltonian (a) and for the same Hamiltonian with all frequencies decreased by a factor of four (b). 

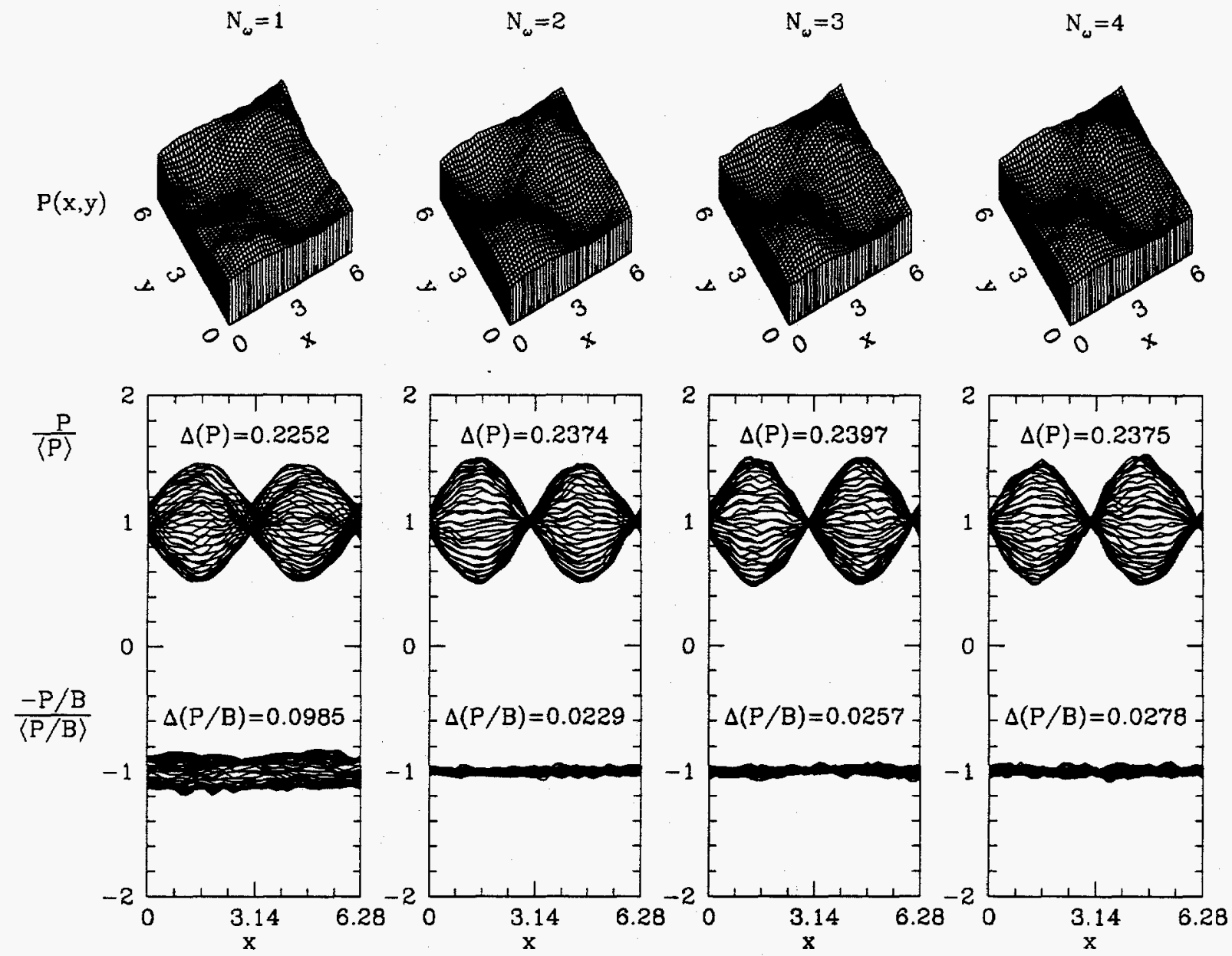

Figure 3: The probability density distribution $P(x, y)$ (top), the vertical cross-sections of the surface of $P$ (middle) and the cross-sections of the surface of $P / B$ (bottom) for the four Hamiltonians.

by the dispersion of $\eta=P / B$,

$$
\Delta(\eta)=\frac{\left\langle(\eta-\langle\eta\rangle)^{2}\right\rangle^{1 / 2}}{\langle\eta\rangle},
$$

and is consistent with the statistical error of $N^{-1 / 2}$, where $N$ is the accumulated number of particle visits to a bin. The particle density in the periodic case $N_{\omega}=1$ deviates from the ergodic (TEP) distribution much more significantly.

\section{Discussion}

The issue of ergodic versus non-ergodic behavior in Hamiltonian systems can be put in the prospective of the relation between the the "strong transitivity" of Birkhoff [1] and Hopf [2], 
on the one hand, and the invariant tori of Kolmogorov [6], Arnold [7], and Moser [8], on the other hand.

The property of strong transitivity of motion $\dot{\mathbf{x}}=\mathbf{v}(\mathbf{x})$ in a finite phase-space domain is defined by the requirement that any invariant set (that is, a set swept by a family of orbits) have either the measure of zero or the measure of the whole domain. In other words, the strong transitivity prohibits against the domain partitioning by impermeable "walls" made up of invariants sets. The property of strong transitivity and the incompressibility of the phase-space flow $\mathbf{v}(\mathbf{x})$ are sufficient for the ergodic behavior [1].

The simplest example of invariant walls is the surfaces of an integral of motion, if one exists. In that case one can consider a reduced phase space confined to one such surface and so on until no more integral constraints remain. Far less trivial is the existence of other invariant sets not related to any global conservation law: the KAM surfaces (KAM tori). Although their presence was proven for Hamiltonian systems sufficiently close to integrable, there is little doubt that remains of KAM tori are present in a general nonintegrable Hamiltonian system. The crucial question is the dimension of the KAM surfaces and their ability to partition the phase-space domain into non-communicating sub-domains. In a conservative $N$-degree-of-freedom system the KAM surfaces are $N$-dimensional [18], and fail to partition the $(2 N-1)$-dimensional iso-energetic manifold if $N<2 N-2$, that is, if $N \geq 3$. This is the case for the Arnold diffusion. Now, if one assumes that there are no invariant sets of dimension higher than that of the KAM tori (excluding the $2 N$-dimensional domain itself), than the onset of the Arnold diffusion is equivalent to strong transitivity, and the ergodicity of the motion follows.

This is exactly this conjecture which appears to be confirmed in our simulation. Indeed, let us rewrite a two-dimensional time-dependent system with a quasiperiodic Hamiltonian $H(x, p, t)$ in a form containing several different "times" $\tau_{i}=\omega_{i} t, i=1,2, \ldots, N_{\omega}$, such that the Hamiltonian $\mathcal{H}(x, p, \tau)=H(x, p, t)$ is $2 \pi$-periodic in each $\tau_{i}$. Introduce $N_{\omega}$ new variables $E_{i}$ satisfying the relation for the total energy $E(t)=\sum \omega_{i} E_{i}$. The choice of $E_{i}$ is not unique, but it can be narrowed by the requirement set by the dynamical equations $\dot{E}_{i}=\partial \mathcal{H} / \partial \tau_{i}$. These equations are consistent because the change of the total energy, $\dot{E}=\sum \omega_{i} \partial \mathcal{H} / \partial \tau_{i}$, duly equals $\partial H(x, p, t) / \partial t$. Now the $2 \mathrm{D}$ time-dependent system can be rewritten as a conservative $2\left(N_{\omega}+1\right)$-dimensional [or $\left(N_{\omega}+1\right)$-degree-of-freedom] system:

$$
\begin{aligned}
\dot{x} & =\partial_{p} K, \quad \dot{p}=-\partial_{x} K \\
\dot{\mathbf{E}} & =\partial_{\tau} K, \quad \dot{\tau}=-\partial_{\mathbf{E}} K,
\end{aligned}
$$

where

$$
K(x, p, \mathbf{E}, \boldsymbol{\tau})=\mathcal{H}(x, p, \boldsymbol{\tau})-\boldsymbol{\omega} \cdot \mathbf{E}
$$

is the new Hamiltonian, whose conserved value is zero. Thus adding a new, incommensurate with old, frequency to the Hamiltonian time dependence is equivalent to adding another degree of freedom. In our case, two or more incommensurate frequencies $\left(N_{\omega} \geq 2\right)$ mean three or more degrees of freedom, where Arnold diffusion takes place and, as far as our simulation shows, the ergodic behavior in the $(x, p)$ plane is observed. 
As a byproduct, the conjecture of Refs. $[9,10]$ that the turbulent electrostatic drift of charged particles in an inhomogeneous magnetic field leads to a biased diffusion and the resulting nontrivial turbulent equipartition density $P(x, y) \propto B(x, y)$ is numerically confirmed for a simple model of quasiperiodic potential. The accuracy of the TEP prediction is found to be within the the statistical sampling error margin. It is expected that this result is exact for a wide class of turbulent (non-quasiperiodic) potentials, which can be considered as a limiting case when the number of the incommensurate frequencies goes to infinity.

\section{Acknowledgments}

This work was supported by the US Department of Energy under grants DE-FG05-80ET53266 and DE-FG05-80ET53088.

\section{References}

[1] G. D. Birkhoff. Proof of the ergodic theorem. Proc. Nat. Acad. Sci., 17:656-660, 1931.

[2] E. Hopf. Statistik der geodätischen Linien in Mannigfaltigkeiten negativer Krümmung. Ber. Verh. Sächs. Akad. Wiss. Leipzig Math. Phys., 91:261-304, 1939.

[3] D. V. Anosov. Geodesic flows on closed Riemannian manifolds of negative curvature. Proc. Steklov Inst. Math., 90:1-235, 1967.

[4] Ya. B. Pesin. Characteristic Lyapunov exponents and smooth ergodic theory. Usp. Mat. Nauk, 32(4):55-112, 1977. English transl.: Russian Math. Surv. 32(4), 55-114 (1977).

[5] A. Katok and K. Burns. Infinitesimal Lyapunov functions, invariant cone families and stochastic properties of smooth dynamical systems. Ergod. Th. \& Dynam. Sys., 14:757$785,1994$.

[6] A. N. Kolmogorov. On the conservation of quasi-periodic motion for a small change in the hamiltonian function. Dokl. Akad. Nauk SSSR, 98:527, 1954. In Russian.

[7] V. I. Arnold. Proof of a theorem of A. N. Kolmogorov on the invariance of quasi-periodic motions under small perturbations of the hamiltonian. Usp. Mat. Nauk (SSSR), 18:91, 1963. [English transl. Russ. Math. Surv. 18, 9 (1963)].

[8] J. Moser. Convergent series expansions for quasi-periodic motions. Math. Ann., 169:163, 1967.

[9] V. V. Yankov. The pinch effect explains turbulent transport in tokamaks. JETP Lett., 60(3):171-176, 1994.

[10] M. B. Isichenko, A. V. Gruzinov, and P. H. Diamond. Invariant measure and turbulent pinch in tokamaks. FRC Report FRCR-452. Submitted to Phys. Rev. Lett., October 1994. 
[11] J. Nycander and V. V. Yankov. Anomalous pinch flux in tokamak driven by the longitudinal adiabatic invariant. Preprint UPTEC 94134 R. Submitted to Phys. Rev. Lett., December 1994.

[12] F. Kang. Difference schemes for Hamiltonian formalism and symplectic geometry. $J$. Comput. Math., 4:279, 1986.

[13] J. Cary. General symplectic integration algorithms through fourth order. Bull. Am. Phys. Soc., 34(9):1927, 1989.

[14] T. G. Northrop. The adiabatic motion of charged particles. Interscience Publishers, New York, 1963.

[15] J. B. Taylor. Equilibrium and stability of plasma in arbitrary mirror fields. Phys. Fluids, $7(6): 767-773,1964$.

[16] M. B. Isichenko. Can computer simulation predict the real behavior of turbulence? Comments on Plasma Phys. Controlled Fusion, 16(3):187-206, 1994.

[17] A. V. Gruzinov, M. B. Isichenko, and J. Kalda. Two-dimensional turbulent diffusion. Zh. Eksp. Teor. Fiz., 97:476-488, 1990. [Sov. Phys. JETP 70, 263-269 (1990)].

[18] V. I. Arnold. Mathematical methods of classical mechanics. Springer, New York, 1978.

\section{DISCLAIMER}

This report was prepared as an account of work sponsored by an agency of the United States Government. Neither the United States Government nor any agency thereof, nor any of their employees, makes any warranty, express or implied, or assumes any legal liability or responsibility for the accuracy, completeness, or usefulness of any information, apparatus, product, or process disclosed, or represents that its use would not infringe privately owned rights. Reference herein to any specific commercial product, process, or service by trade name, trademark, manufacturer, or otherwise does not necessarily constitute or imply its endorsement, recommendation, or favoring by the United States Government or any agency thereof. The views and opinions of authors expressed herein do not necessarily state or reflect those of the United States Government or any agency thereof. 